\title{
Western Australia Atropine for the Treatment of Myopia (WA- ATOM) study: rationale, methodology, and participant baseline characteristics
}

\author{
Samantha S-Y Lee PhD, ${ }^{1}$ David A. Mackey MD FRANZCO, ${ }^{1,2,3}$, Gareth Lingham \\ MOrth, ${ }^{1}$ J ulie M. Crewe BSc, ${ }^{1}$ Fred K. Chen PhD FRANZCO, ${ }^{1,4}$ J ason Charng PhD, ${ }^{1}$ \\ Fletcher Ng MBBS, ${ }^{1}$ I an Flitcroft MD, ${ }^{5}$ J ames J. Loughman PhD, ${ }^{6}$ Augusto Azuara- \\ Blanco MD, ${ }^{7}$ Nicola S. Logan PhD, ${ }^{8}$ Christopher J. Hammond MD, ${ }^{9}$ Audrey Chia PhD \\ FRANZCO, ${ }^{10}$ D. Tan Tai Truong MPS ${ }^{11}$ and Antony Clark PhD FRANZCO ${ }^{1}$
}

${ }^{1}$ Centre for Ophthalmology and Visual Sciences (incorporating Lions Eye Institute), University of Western Australia, Perth, WA, Australia ${ }^{2}$ Centre for Eye Research Australia, University of Melbourne, Royal Victorian Eye and Ear Hospital, VIC, Australia

${ }^{3}$ School of Medicine, Menzies Research Institute Tasmania, University of Tasmania, TAS, Australia

${ }^{4}$ Department of Ophthalmology, Royal Perth Hospital, Perth, WA, Australia ${ }^{5}$ Department of Ophthalmology, Mater Misericordiae University Hospital, Dublin, Ireland

${ }^{6}$ Centre for Eye Research I reland, School of Physics, Clinical and Optometric Sciences, Technological University Dublin, Dublin, I reland ${ }^{7}$ School of Medicine Dentistry, and Biomedical Science, Queen's University Belfast, Ireland ${ }^{8}$ School of Life \& Health Sciences, Aston University, Birmingham, United Kingdom This is the author manuscript accepted for publication and has undergone full peer review but has not been through the copyediting, typesetting, pagination and proofreading process, which may lead to differences between this version and the Version of Record. Please cite this article as doi: 10.1111/ceo.13736

This article is protected by copyright. All rights reserved. 
${ }^{9}$ Department of Twin Research and Genetic Epidemiology, King's College London, St. Thomas' Hospital, London, United Kingdom

${ }^{10}$ Singapore National Eye Centre, Singapore; Singapore Eye Research Institute, Singapore

${ }^{11}$ Oxford Compounding, North Perth, WA, Australia

Correspondence: Dr Antony Clark, Lions Eye Institute, Centre for Ophthalmology and Visual Science, University of Western Australia, Perth, WA 6009, Australia Email: AntonyClark@lei.org.au

Short running title: Atropine treatment of myopia

Received 16 J anuary 2020; accepted 18 February 2020

Funding sources / Financial disclosure: The WA-ATOM study is funded by a Telethon-Perth Children's Hospital Research Fund, the Ophthalmic Research Institute of Australia, a University of Western Australia (UWA) Health and Medical Science Early Career Researcher Small Grant Award, a Healy's Research Collaboration Award, and a UWA Research Collaboration Award. The funding organizations had no role in the design or conduct of this research.

Conflict of interest: None 


\section{ABSTRACT}

I mportance: Atropine eyedrops are a promising treatment for slowing myopia progression in East Asian children. However, its effects on children in Australia, including those of non-Asian background, have not been well-studied.

Background: The Western Australia Atropine for the Treatment of Myopia (WAATOM) study aims to determine the efficacy and long-term effects of low-dose atropine eyedrops in myopia control. This paper describes the study rationale, methodology, and participant baseline characteristics.

Design: Single-centre, double-masked, randomised controlled trial

Participants: Children (6-16 years) with spherical equivalent $\leq-1.50 \mathrm{D}$ in each eye, astigmatism $\leq 1.50 \mathrm{D}$, and myopia progression by $\geq 0.50 \mathrm{D} /$ year.

Methods: Enrolled children were randomly assigned 2:1 to receive $0.01 \%$ atropine or placebo eyedrops. Participants are examined every six months during first three years of the study (2-year treatment phase followed by a 1-year washout phase), and then at a 5-year follow-up (two years after the end of the washout phase).

Main outcome measures: Annual progression rate of myopia and axial length, tolerability to eyedrops, and incidence and severity of unwanted effects.

Results: Out of 311 children who were referred, 242 were suitable for study participation, and 153 were subsequently enrolled. The baseline characteristics of enrolled participants are presented.

Conclusions and relevance: Outcomes of the WA-ATOM study will inform on the efficacy, tolerability, safety, and long-term effects of low-dose atropine eyedrops in myopia control in Australian children. The impact of ocular sun exposure, iris colour, and parental myopia on the efficacy of low-dose atropine will also be assessed.

Keywords: Atropine eyedrops, myopia, myopia control, myopia treatment 


\section{I NTRODUCTION}

Topical atropine eyedrops have been postulated as a potential intervention for slowing the progression of myopia for over a century. ${ }^{1-5}$ However, it was not until the landmark Atropine for the Treatment of Myopia (ATOM1 and ATOM2) studies in Singapore ${ }^{6-10}$ that the interest in atropine eyedrops for myopia control escalated. These ATOM studies, conducted in the 2000s to early 2010s, were the first welldesigned, randomised controlled trials (RCTs) that demonstrated atropine eyedrops are effective for slowing myopia progression in children of East Asian descent.

A surprising finding that arose from the ATOM2 study ${ }^{6-8}$ was that even a very low concentration of atropine $(0.01 \%)$ seem to slow myopia progression. In children who used $0.1 \%, 0.5 \%$, or $1.0 \%$ atropine eyedrops for 2 years, a "rebound effect" was noted after treatment cessation. However, this effect was not observed in those using $0.01 \%$ atropine. ${ }^{7}$ At the end of the 3-year ATOM trials (including the 2-year treatment and 1-year washout phases), myopia in children on low-dose (0.01\%) atropine progressed by only -0.72 dioptres (D) of spherical equivalent and $+0.58 \mathrm{~mm}$ of axial length from baseline. ${ }^{7,10}$ These rates are similar to those in children who used relatively higher doses of atropine $(0.1 \%$ or $0.5 \%)$, which resulted in progression in spherical equivalent by $-1.04 \mathrm{D}$ and in axial length by $0.60 \mathrm{~mm}$ or more. ${ }^{7,}{ }^{10}$ While the best long-term control of axial length was observed with a higher dose of atropine (1.0\%), with only a $0.3 \mathrm{~mm}$ increase over 3 years, ${ }^{10}$ higher doses of atropine are associated with higher incidence of unwanted effects such as allergic or hypersensitivity reactions, discomfort, photophobia, and reduced accommodation. ${ }^{6,9}$ For example, the ATOM studies reported that only after 2 weeks of high-dose atropine use ( 0.1 to $1.0 \%$ ), amplitude of accommodation was reduced by 11 to $14 \mathrm{D}$, compared to only a 5D reduction in those using atropine $0.01 \%$. This led the ATOM researchers to conclude that low-dose atropine $(0.01 \%)$ may have the optimal balance between efficacy and safety or tolerability. ${ }^{6}$ 
It should be noted that the ATOM2 study did not have a placebo-control group; the $0.01 \%$ was intended as a control for comparison with the higher concentrations of eyedrops. To date, there are very few studies that have directly evaluated low-dose atropine against a placebo. One high-quality published trial that is currently addressing this is the low-concentration atropine for myopia progression (LAMP) study ${ }^{11}$ in Hong Kong. The LAMP study reported that $0.05 \%$ atropine had the best efficacy in myopia control during the first two years of treatment, compared with $0.025 \%$ or $0.01 \%$ atropine (approximate rate of change in spherical equivalent: $-0.27 \mathrm{D} /$ year, $-0.45 \mathrm{D} /$ year, and $-0.55 \mathrm{D} /$ year, respectively. ${ }^{11,}{ }^{12}$ While a placebocontrol group was initially included in the LAMP study, at the end of the first year, participants who received a placebo switched to receive $0.05 \%$ atropine for the rest of the 5 -year trial. ${ }^{12}$ Thus, there remains a need for a longer-term placebo-controlled RCT.

\subsection{Study rationale}

Many studies on atropine control of myopia have been conducted in East Asian populations, which have the highest prevalence of myopia. ${ }^{13}$ Even though Australia currently has a relatively low rate of myopia, it is predicted to increase from approximately $20 \%$ to $55 \%$ between the years 2000 and 2050 in the Australasia regions. ${ }^{13}$ Addressing myopia in Australia and other Western countries is therefore also a priority.

Despite the successful ATOM trials in Singapore, we cannot assume that their findings can be directly extrapolated to other populations, especially Australia, for two major reasons. First, Australia is fast becoming a multi-ethnic country comprising children of Caucasian, East Asian, South Asian, and mixed descent. Given that the duration and magnitude of action of mydriatic eyedrops depends upon the amount of iris pigmentation, ${ }^{14,} 15$ the effectiveness and safety of atropine eyedrops are expected to differ between children of East Asian and those of other ethnicities. 
While a retrospective study ${ }^{2}$ in California did report that low-dose atropine reduced myopia progression rates in children of various backgrounds, including Asians, Caucasians, Hispanics, and African Americans, the study was non-randomised and the control group did not receive a placebo. Therefore, there remains the need for well-designed RCTs on low-dose atropine for myopia control in non-Asian populations. Second, there are differences in lifestyle between children in Singapore and Australia. Children in Australia tend to spend significantly more time outdoors compared to those in Singapore ${ }^{16,17}$ and reduced time spent outdoors is a wellestablished risk factor for myopia. ${ }^{16}$

The Western Australia Atropine for the Treatment of Myopia (WA-ATOM) study aims to evaluate the efficacy and safety of low-dose $(0.01 \%)$ atropine in reducing myopia progression in an Australian context. The WA-ATOM study is divided up into three phases: (1) a 2-year treatment phase, (2) a 1-year washout phase, and (3) a 2-year "parents' choice" phase. The first two phases were designed to test the hypotheses that (1) low-dose atropine eyedrops $(0.01 \%)$ is a safe, tolerable, and efficient method of controlling myopia progression in comparison to a placebo and (2) there is minimal "rebound" myopia progression effect for at least one year after ceasing application of $0.01 \%$ atropine eyedrops after long-term use (two years). The third phase of the study (parents' choice) serves to assess a longer-term effect of atropine eyedrop use, as well as explore parents' choices of myopia treatment method after they have been unmasked to their children's treatment allocation.

Data from the WA-ATOM study will be combined and analysed with those from two other trials underway in Ireland (Myopia Outcome Study of Atropine in Children [MOSAI C]) ${ }^{18}$ and in the United Kingdom (Children Atropine for Myopia Progression [CHAMP-UK] ${ }^{19}$ to form a large meta-analysis on atropine treatment in Western populations. The protocols for the CHAMP-UK and MOSAIC trials have been planned with respect to the WA-ATOM study, thus enabling merger of anonymised 
individual participant data. The prospective nature of this meta-analysis will be advantageous over retrospective analyses and also increase the power to allow subgroup analyses. In this paper, we describe the detailed methodology and participant baseline characteristics of the WA-ATOM study.

\section{METHODS}

\subsection{Study design}

This single-centre, double-masked RCT has two treatment arms: $0.01 \%$ atropine (intervention) and placebo (control) eyedrops (2:1 allocation). An uneven allocation was used to increase willingness of participation and improve recruitment rates. In phase 1 of the study (treatment phase), participants use their allocated eyedrops on a nightly basis and are monitored every six months. This is followed by a 1-year washout phase during which no eyedrops are used, with continued 6monthly monitoring of participants after treatment cessation. Participants are unmasked at the end of the washout phase, after which, these participants are essentially "discharged" from the study, and are informed to continue to routinely see their usual eye care provider (optometrist or ophthalmologist). Participants return for a final eye examination two years after the unmasking (at the 5-year mark). The purpose of this follow-up at the 5-year mark is to explore the participant's choices of treatment after they have learnt of the study outcomes, as well as to explore the longer-term effects of previous nightly atropine eyedrop use in the treatment group. As the decision to extend the study by two years was made after all participants have been enrolled and informed that it was a 3-year trial, , participants are free to decide on their own method of myopia control (including no treatment, multifocal spectacles/contract lenses, orthokeratology, or atropine eyedrops prescribed privately) after they are unmasked, with consultation with their own eye care provider. 


\subsection{Sample size considerations}

In the ATOM2 study, the $0.01 \%$ concentration reduced myopia progression by $60 \%$ compared to the ATOM1 placebo group (-0.49D/year vs. $-1.20 \mathrm{D} /$ year). As it has been suggested that atropine may be less effective in controlling myopia in Caucasians compared to Asians, ${ }^{20}$ we made a conservative estimate of the effect size, aiming to detect at least a $40 \%$ difference in myopia progression rate between the treatment and control groups. Based on this effect size, a type I error of 0.05, 90\% power, and the 2:1 allocation ratio, 58 participants in the treatment group and 29 in the control group are needed. Assuming an attrition rate of 15\%, at least 103 participants need to be enrolled. We increased this to 150 participants (100 in treatment group and 50 in control group) to account for potential missing data.

\subsection{Participant recruitment}

Recruitment began in May 2017. The trial was promoted to the optometry and ophthalmology communities in Western Australia through professional networking, presentations, and similar events. The study was advertised in local newspapers and the Lions Eye Institute website. Referrals from eyecare practitioners were sent directly to the investigators, who then screened the refraction history to determine the documented amount of myopia progression over the previous 12 months. Based on the information on the referral, the investigators then make a preliminary decision on study eligibility (Table 1). The parents or guardians of potential participants were then contacted via email or telephone and given a detailed explanation of the study. The participant information sheet and visit appointment confirmation were emailed to the parent/guardian of potential participants when they were booked in for their initial visit. Formal written informed consent was obtained at the start of the initial visit followed by baseline assessments 
(Table 2), during which the final eligibility of the participant was confirmed (i.e. met all inclusion criteria; Table $\mathbf{1}$ ). 
Table 1: Inclusion and exclusion criteria

\section{Inclusion criteria}

- Age 6 to 16 years old (inclusive) at the time of enrolment

- Cycloplegic spherical equivalent of $\leq-1.50 \mathrm{D}$ in each eye ${ }^{\dagger}$

- Documented myopia progression over previous 12 months in either eye

- Astigmatism not more than $1.50 \mathrm{DC}^{\dagger}$

- Anisometropia not more than $1 \mathrm{D}^{\dagger}$

- Best-corrected visual acuity (logarithm of minimum angle of resolution, logMAR) of 0.2 (equivalent Snellen fraction $=6 / 9$ ) or better in each eye ${ }^{\dagger}$

- Willing and able to provide details of parents' country of origin

- Able to provide appropriate parental or guardian consent

Exclusion criteria ${ }^{\S}$

- Severe developmental delay that renders them unable to complete eye tests

- Ocular comorbidities including, but not limited to, amblyopia, glaucoma, aphakia, strabismus, pseudophakia, active or current uveitis, keratoconus, and severe ocular surface disease

- Systemic comorbidities including, but not limited to, cardiac or respiratory disease, and connective tissue disease (e.g. Marfan Syndrome)

- Previously received atropine therapy for amblyopia or myopia progression

- Use of orthokeratology lenses within the past 3 months

${ }^{\dagger}$ As documented by participants' own optometrists/ophthalmologists and confirmed

during the baseline visit; ${ }^{*}$ As documented by participants' own

optometrists/ophthalmologists; 's Determined using parent/guardian-reported

information, referral from medical or health practitioner, and clinical observations

during baseline assessment 
Table 2: Summary of study protocol at each visit, ordered according to test sequence

\begin{tabular}{|c|c|c|c|c|c|c|c|c|}
\hline \multirow[b]{2}{*}{ Study measures } & \multicolumn{4}{|c|}{ Treatment phase } & \multicolumn{2}{|c|}{$\begin{array}{c}\text { Washout } \\
\text { phase }\end{array}$} & \multirow{2}{*}{$\begin{array}{c}\text { “Parents' } \\
\text { choice" } \\
\begin{array}{c}60- \\
\text { month }\end{array}\end{array}$} & \multirow{2}{*}{$\begin{array}{c}\text { Parental } \\
\text { eye }\end{array}$} \\
\hline & I nitial & $\begin{array}{c}2- \\
\text { week }\end{array}$ & $\begin{array}{l}\text { 6-, 12-, } \\
\& \quad 18- \\
\text { month }\end{array}$ & $\begin{array}{c}\text { 24- } \\
\text { month }\end{array}$ & $\begin{array}{l}\text { 30- } \\
\text { month }\end{array}$ & $\begin{array}{c}\text { 36- } \\
\text { month }\end{array}$ & & \\
\hline a) Presenting VA & $\sqrt{ }$ & $\sqrt{ }$ & $\sqrt{ }$ & $\sqrt{ }$ & $\sqrt{ }$ & $\sqrt{ }$ & $\sqrt{ }$ & $\sqrt{ }$ \\
\hline b) Autorefraction & $\sqrt{ }$ & $\sqrt{ }$ & $\sqrt{ }$ & $\sqrt{ }$ & $\sqrt{ }$ & $\sqrt{ }$ & $\sqrt{ }$ & $\sqrt{ }$ \\
\hline c) NPA & $\sqrt{ }$ & $\sqrt{ }$ & $\sqrt{ }$ & $\sqrt{ }$ & $\sqrt{ }$ & $\sqrt{ }$ & $\sqrt{ }$ & \\
\hline d) Stereoacuity & $\sqrt{ }$ & & & $\sqrt{ }$ & & $\sqrt{ }$ & $\sqrt{ }$ & \\
\hline e) Pupillometry & $\sqrt{ }$ & $\sqrt{ }$ & $\sqrt{ }$ & $\sqrt{ }$ & $\sqrt{ }$ & $\sqrt{ }$ & $\sqrt{ }$ & \\
\hline f) Ocular biometry & $\sqrt{ }$ & & $\sqrt{ }$ & $\sqrt{ }$ & $\sqrt{ }$ & $\sqrt{ }$ & $\sqrt{ }$ & $\sqrt{ }$ \\
\hline g) Eye colour documentation & $\sqrt{ }$ & & & & & & & \\
\hline h) CUVAF & $\sqrt{ }$ & & & $\sqrt{ }$ & & $\sqrt{ }$ & $\sqrt{ }$ & \\
\hline i) Tonometry & $\sqrt{ }$ & $\sqrt{ }$ & $\sqrt{ }$ & $\sqrt{ }$ & $\sqrt{ }$ & $\sqrt{ }$ & $\sqrt{ }$ & \\
\hline j) Cycloplegic eye drop instillation ${ }^{\ddagger}$ & $\sqrt{ }$ & $\sqrt{ }$ & $\sqrt{ }$ & $\sqrt{ }$ & $\sqrt{ }$ & $\sqrt{ }$ & $\sqrt{ }$ & $\sqrt{ }$ \\
\hline k) Fundus photography & $\sqrt{ }$ & & & $\sqrt{ }$ & & $\sqrt{ }$ & $\sqrt{ }$ & \\
\hline I) Choroidal thickness measurement ${ }^{\S}$ & $\sqrt{ }$ & & & $\sqrt{ }$ & & $\sqrt{ }$ & $\sqrt{ }$ & \\
\hline m) Anterior segment topography & $\sqrt{ }$ & & & $\sqrt{ }$ & & $\sqrt{ }$ & $\sqrt{ }$ & $\sqrt{ }$ \\
\hline n) Post-cycloplegic autorefraction & $\sqrt{ }$ & $\sqrt{ }$ & $\sqrt{ }$ & $\sqrt{ }$ & $\sqrt{ }$ & $\sqrt{ }$ & $\sqrt{ }$ & \\
\hline $\begin{array}{l}\text { o) Self-administered questionnaire on } \\
\text { eyedrop use }\end{array}$ & & $\sqrt{ }$ & $\sqrt{ }$ & $\sqrt{ }$ & & & & \\
\hline
\end{tabular}


CUVAF = conjunctival ultraviolet autofluorescence; ETDRS= Early treatment of diabetic retinopathy study; NPA= near point of accommodation; $\mathrm{VA}=$ visual acuity; ${ }^{\dagger}$ conducted in each biological parents where possible, at any time during the study period; ${ }^{\ddagger}$ one to three drops instilled depending on the amount of dilation achieved; § using the enhanced depth imaging mode on an optical coherence tomography; " includes questions on child's tolerability and attitude towards eyedrop use 


\subsection{Intervention and randomisation}

At the first visit, each enrolled child picked a unique randomisation number from a jar and each number represented allocation to either intervention arm: atropine sulphate $0.01 \%$ (treatment) or placebo eyedrops. In the first three years of the study, both the examiner and participants are unaware of which arm the randomisation numbers corresponded to and thus were masked to the intervention received. Only the compounding pharmacists maintain the randomisation list. Both the atropine and placebo are packaged in identical bottles labelled with only the participant's name and expiry date. Participants are unmasked at the end of the first three years of the study by an investigator who is not involved in examining the patient or study data analysis.

The eyedrops are packaged in 10 millilitre bottles and preserved with $0.01 \%$ benzalkonium chloride. Participants were instructed to instil one drop into each eye every night. An offsite pharmacy (Oxford Compounding, North Perth, WA, Australia) compounds and dispenses the eyedrops. Each bottle is stored in an amber plastic vial for protection against photodegradation. Only a maximum of three bottles are dispensed each time and to further preserve the integrity of the eyedrops, participants are instructed to keep the bottles in the refrigerator and to use a fresh bottle every month. To monitor adherence to treatment, each child is given a calendar in which they mark the days that the eyedrops are instilled.

\subsection{Follow-up visits}

Following the initial assessments, participants attended a 2-week follow-up visit (baseline 2) as per the Singapore ATOM studies. ${ }^{6,9}$ Subsequently, follow-up visits are on a 6-monthly basis up to the first 3 years of the study ( 2 year treatment and 1 year washout). The eye tests conducted during each study visit are detailed below and outlined in Table 2. 


\subsection{Parents' eye testing}

To evaluate the possible influence of parental myopia on the efficacy of atropine treatment, biological parents underwent a refractive screening (Table 2). Parents were additionally asked if they had undergone laser refractive surgery, and if so, what their estimated refractive errors were prior to surgery.

\subsection{Study measurements}

- Visual acuity (VA): distance and near VAs are measured monocularly using EDTRS logMAR charts (distance and near charts respectively). Participants wear their habitual spectacle correction for both distance and near and are encouraged to read the smallest line they can see. For each eye and testing distance, pinhole VA is additionally measured regardless of the level of their presenting VA. VA is scored on a letter-by-letter basis and recorded in logMAR notation. ${ }^{21}$

- Autorefraction/autokeratometry: Autorefraction (Nidek ARK-510A, NIDEK Co. Ltd, J apan) is used to measure refractive error before cycloplegia and at least 20 minutes after instillation of 1 to 3 drops of cyclopentolate $1 \%$. Refractive error is recorded in minus-cylinder form and the spherical equivalent is determined as the spherical component plus half of the cylindrical component.

- Vertometry. Participants' presenting spectacle lenses using an auto lensmeter (Nidek LM-7P, NIDEK Co. Ltd, Japan). Spectacles are measured at the baseline visit and repeated at follow-ups if participants obtain a new pair in between visits.

- Amplitude of accommodation. Near point of accommodation (NPA) is measured binocularly using a Royal Air Force (RAF) Rule (Good-Lite Company, Elgin, Illinois, United States of America). Starting from a viewing distance of 30 centimeters $(\mathrm{cm})$, a target (an N8-sized line of 5 words) is presented to the participant. Participants are encouraged to maintain visual clarity of the target as it is gradually moved closer to the participant. When the participant reports that they are no longer able to see the N8 words clearly, the target is then slowly moved 
backwards until the participant reports that they are able to see the words clearly again, the distance at which is recorded as the NPA $(\mathrm{cm})$..

- Stereoacuity. a Titmus Stereo Fly Test (Good-Lite Company, Elgin, Illinois, United States of America). The graded circle test portion is used to determine stereoacuity, which has a measurement range of 800 to 40 seconds of arc (arcsec).

- Pupillometry: Pupillary size and light reflex in both eyes are evaluated using a NPi-200 digital pupillometer (NeurOptics Inc., Laguna Hills, California, United States of America). The device consists of a colour LCD screen that displays the measurements, a built-in infrared digital camera that records pupil reactions, and light-emitting diodes (LEDs) surrounding the camera which emit visible white light at an intensity of 50 microwatts $(\mu \mathrm{W})$. At the start of each measurement, the LEDs flash the light for 0.8 seconds $(\mathrm{s})$ to stimulate pupil constriction. Pupil reactivity is recorded for an additional $4.2 \mathrm{~s}$ to monitor the dilation phase, totaling a measurement time of $5 \mathrm{~s}$ per eye. Measurements are taken monocularly, and participants are instructed to fixate on a dim red target 3 meters away with the nonmeasured eye. Optical correction is not used so as to not elicit any accommodative pupil constriction. All measurements are taken in complete darkness apart from the red fixation light to achieve maximum dilation. Readings from the pupilometer include the minimum and maximum pupil diameters, percentage decrease in pupil size (relative to maximum size), latency of constriction, velocity of constriction, and velocity of dilation.

- Ocular biometry. axial length (AL), white-to-white corneal diameter, keratometry, and anterior chamber depth are measured using an IOLMaster V5 (Carl Zeiss Meditec AG, Jena, Germany) prior to cycloplegia. The average of at least five AL measurements is recorded, excluding erroneous measurements (e.g. extremely high or low values that may be caused by subject movement) and those that fall outside one standard deviation of the average. Two to three white-to-white measurements are taken and the median of these is recorded. 
- Eye colour. high resolution and close-up photographs of the participants' anterior eyes are taken, prior to pupil dilation, using a Nikon D1000 digital camera (Tokyo, J apan) with a $105 \mathrm{~mm} 147 \mathrm{f} / 2.8$ Micro Nikkor lens (Nikon, Melville, New York, USA). Categorization of eye colour is based on a previously published scheme. 22

- Conjunctival ultraviolet autofluorescence (CUVAF): CUVAF refers to areas on the bulbar conjunctiva with actinic damage that fluoresces under ultraviolet light, which provides an objective measure of ocular sun exposure. ${ }^{23}$ CUVAF images of the nasal and temporal bulbar conjunctiva of each eye are taken using a Nikon D100 digital camera (Tokyo J apan) with a 105 mm 147 f/2.8 Micro Nikkor lens (Nikon, Melville, New York, USA) fitted with a UV filter (B+W 486 UV IR filter, Schneider Kreuznach, Bad Kreuznach, Rhineland-Palatinate, Germany). Two external electronic Metz 36C-2 flashes (Metz, Zirndorf, middle Franconia, Germany) are fittedwith UV transmission Wratten glass filters resulting in a peak transmission of $365 \mathrm{~nm}$ (range 300 to $400 \mathrm{~nm}$ ). Images are taken in a dark room to ensure that only ultraviolet autofluorescence is recorded. The total area of CUVAF for each participant is quantified offline using Matlab 2017b.

- Tonometry. intraocular pressure is measured prior to cycloplegia using an ICare rebound tonometer (I care TAOli Tonometer, I care Finland Oy, Helsinki, Finland).

- Cycloplegia. participants' eyes are first topically anaesthetized (one drop proxymetacaine hydrochloride $0.5 \%$ ) prior to instillation of cyclopentolate $1 \%$. One to three drops of cyclopentolate are instilled depending on the amount of dilation achieved.

- Fundus photography. An Optos California ICG (Optos, Dunfermline, United Kingdom) is used to capture fundus images of each eye. The system is able to capture up to a $200^{\circ}$ view of the fundus in an undilated eye with a resolution of 20 $\mu \mathrm{m}$. 
- Macular choroidal thickness measurements. cross-sectional spectral-domain optical coherence tomography scans of the central retina are obtained using the enhance depth imaging mode (Spectralis HRA + OCT, Heidelberg Engineering, Heidelberg, Germany). Two horizontal and two vertical B-scans centred on the fovea are taken for each eye, with each scan spanning $8.6 \mathrm{~mm}\left(\sim 30^{\circ}\right)$. To maximize image quality, an automated real-time tracker is used and the average of 100 frames is recorded for analysis. EDI scans are exported and the choroidal thicknesses are measured offline.

- Anterior segment topography: an Oculus Pentacam (software version 6.08r27; OculusOptikgerate $\mathrm{GmbH}$, Wetzlar, Germany) is used to obtain tomography images of the corneal, anterior chamber, and crystalline lens. The main parameters of interest obtained are corneal thickness and crystalline lens thickness.

- Quality of life: the parents or guardians of participants are asked to complete a questionnaire at each follow-up visit. The questionnaire consists of 18 questions, most of which focus on the child's tolerance to the eyedrops. Responses are on a scale from "strongly agree" to "strongly disagree" and include a "not applicable" response. A comment box is provided at the end of the questionnaire to allow the parents or guardians to insert any additional quality of life related effects that are not covered in the 18 questions.

\subsection{Ethics and safety monitoring}

The trial is registered on the Australia New Zealand Clinical Trials Registry (registration number ACTRN12617000598381) and the study is being conducted in accordance with the tenets of the Declaration of Helsinki. Ethics approval was obtained from the Human Research Ethics Committees at the University of Western Australia and the Perth Children's Hospital (formerly Princess Margaret Hospital for Children). The placebo and treatment eyedrops were approved by the Therapeutics Goods Administration, Department of Health, Australia. The nature of the study was 
fully explained to the participants (child and parent/guardian), and verbal assent was obtained from the participating child before written informed consent was obtained from the parent/guardian. An independent Data and Safety Monitoring Board oversees the study and advises on the suitability of the study to continue based on its progress and participant safety (incidence and types of adverse events).

\subsection{Outcome measures}

The primary outcome measures are the annual changes in spherical equivalent (post-cycloplegia; D/year) and axial length ( $\mathrm{mm} /$ year). Secondary measures include changes in subfoveal choroidal thickness, corneal curvature, corneal thickness, and crystalline lens thickness. Measures of atropine tolerability and safety include amplitude of accommodation, pupil reactions and size, and incidence of adverse or unwanted effects.

\subsection{Statistical analysis}

All analyses will be conducted on an intention-to-treat basis. Demographic and baseline data will be tabulated by treatment arm using appropriate summary statistics. Treatment comparison will be made using t-tests and Wilcoxon rank sum tests for continuous variables with parametric and non-parametric distributions, respectively. Categorical variables will be compared between the treatment groups using Fisher's Exact tests.

At the end of the 24-month treatment phase, ocular data from both eyes will be pooled to give a single mean value prior to analysis using vector-generalised additive models (VGAM) to account for potential skewness and leptokurtosis in the ocular outcomes. Robust standard errors for clustered data will be employed and all VGAM regression models will include treatment group and adjustments for possible confounding factors, including age, CUVAF area, eye colour, parental myopia, and baseline ocular measurements. Longitudinal ocular parameter data will be analysed 
using linear mixed effects models as this method which addresses potential confounders over time, missing data at random, and the within-subject correlation between the two eyes, and it is suited for evaluating individual effects. ${ }^{24,}{ }^{25} \mathrm{To}$ enhance power for subgroup analysis (notably ethnic groups), single-subject prospective meta-analysis will be conducted together with the data collected from the CHAMP-UK study and MOSAIC. Analyses will be performed using the opensource R statistical software (2017 The R Foundation for Statistical Programming, https://www.r-project.org/ [open-source]).

\section{RESULTS}

Recruitment concluded in December 2019. A total of 312 children were referred for the study from optometrists ( $n=268 ; 85.9 \%$ ) or ophthalmologists $(n=11 ; 3.5 \%)$ in private practices, and a general practitioner $(n=1 ; 0.3 \%)$. Thirtytwo parents (10.2\%) contacted the study coordinator directly; the majority of these sought interest in the study after seeing the advertisement in a local newspaper ( $\mathrm{n}=$ 26). One parent became aware of the study from word-of-mouth; while another 5 parents already had a child enrolled in the study and self-referred their other child.

Out of the 312 children referred, 62 (19.8\%) were not suitable for the trial based on their refraction or refractive history provided by their optometrist. Of the remaining 250 children, the parents of 159 of them (63.6\%) gave consent for screening, and 158 were determined to be eligible. Five parents decided not to enroll their children in the study, leaving a total of 153 children enrolled and randomised.

\subsection{Participant baseline characteristics}

Mean age of randomised participants was $11.5 \pm 2.7$ years. The sample consisted of 65 boys ( $42.8 \%)$. About half of the participants are of European background ( $n=75 ; 49.0 \%$ ), with the rest of the cohort comprising children of East Asian ( $n=27 ; 17.6 \%)$, South Asian ( $n=33 ; 21.6 \%)$, other or mixed ethnicities $(n=18$; 
11.7\%). The median spherical equivalent (cycloplegic refraction) was -3.39D (IQR=2.63 to -4.25$)$, and the median rate of progression prior to entering the trial was $0.95 \mathrm{D} /$ year ( $\mathrm{QR}=-0.64$ to $-1.28 \mathrm{D} /$ year). The parent-reported age at myopia diagnosis ranged from 3 to 14 years old (median $=8$ years [IQR=6 to 10 years]). Forty-seven participants ( $30.7 \%$ of sample) had no myopic parents, while 64 $(41.8 \%)$ and 40 (26.1\%) participants had one and two myopic parent(s), respectively. Parental myopia information was not available for two participants as these children were adopted.

\section{DISCUSSION}

The prevalence of myopia is increasing worldwide, ${ }^{13}$ hence effective and well tolerated approaches to stem this tide are needed to prevent the serious complications that result from high myopia. Several methods have been proposed to slow myopia progression, including optical methods such as multi- or bifocal lenses, peripheral defocus modifying lenses, and orthokeratology, as well as structured outdoor time at school. ${ }^{26}$ The optical interventions are based on the hypothesis that myopia progresses as a result of hyperopic defocus at the retina, which triggers axial growth. ${ }^{27}$ Therefore, it has been postulated that by using optical lenses, myopic defocus can be induced at the central retina (e.g. with the use of progressive spectacle lenses) or at the peripheral retina (e.g. orthokeratology, peripheral defocus modifying lenses), thereby slowing axial growth and myopia progression. However, optical methods have only been moderately effective at best in myopia control. In Huang et al.'s meta-analysis ${ }^{26}$ comparing various proposed myopia interventions, optical methods were estimated to reduce refractive error and AL change only by 0.09 to $0.33 \mathrm{D} /$ year and 0.04 to $0.15 \mathrm{~mm} /$ year, relative to wearing single-vision spectacles. 
Pharmacological methods, on the other hand, have shown more promise in myopia control, particularly atropine, which Huang et al. ${ }^{26}$ estimated a slowing in myopia progression by more than $0.50 \mathrm{D} /$ year and axial growth by $0.21 \mathrm{~mm} /$ year compared to a placebo or using single-vision lenses. However, all of the wellconducted atropine studies, included those in the meta-analysis ${ }^{26}$ were in East Asian populations. ${ }^{6,}$, 28,29 To date, there is no well-conducted RCTs in Western countries.

The relatively low rates of myopia in Western countries might be a reason for the lack of RCTs on treatment methods in these populations. In Australia, the low prevalence of myopia has been attributed to the outdoor lifestyle of the population, ${ }^{16,} 30,31$ which is a well-established protective factor against myopia. ${ }^{32}$ As a result, studies in Asia have started evaluating whether increasing time spent outdoors can impact myopia, and these have been met with some success. ${ }^{33,} 34$ The "light-dopamine hypothesis" suggests that exposure to high levels of light (typically outdoor levels of light) stimulates an increased release of dopamine from the retina, inhibiting axial elongation and myopia progression. ${ }^{32}$ This begs the question of whether Australian residents would even benefit (or perhaps benefit more) from atropine treatment given their longer hours of sunlight exposure relative to those who live in Asia.

\subsection{Atropine's mechanism of action}

While it was previously thought that atropine exerts its anti-myopiagenic effects through inhibiting accommodation via the muscarinic receptors in the ciliary body, this hypothesis has largely been rejected. Atropine has also been shown to be effective in inhibiting experimentally induced myopia in chicks, an animal model in which accommodation is primarily mediated by nicotinic receptors. ${ }^{35}$ Furthermore, other cycloplegic eyedrops have shown little efficacy in reducing myopia progression. ${ }^{26}$ Mechanisms that have been proposed more recently include binding to the muscarinic receptors in the sclera, which interferes with scleral growth; ${ }^{36}$ 
reducing Gamma-aminobutyric acid levels which modulate eye growth; 37,38 and binding to muscarinic receptors on "non-specific" retinal cells, inducing dopamine release. $^{39}$

The latter theory ${ }^{39}$ led us to hypothesise that atropine might be less effective in individuals with myopia who already spend more time outdoors (i.e. children in Australian relative to those in Singapore) ${ }^{16,}{ }^{17}$ In a meta-analysis, ${ }^{20}$ atropine was suggested to be less effective in Caucasian populations than in Asians. While a likely reason for this outcome is that the meta-analysis included only a small number of studies with Caucasian children (none of which had a placebo-control group), as well as the relatively slower myopia progression rates in Caucasian children, we suspect that the differences in time spent outdoors could also explain the varying effects of atropine between individuals or populations. Another possible reason that atropine may not be as effective a myopia treatment in a Caucasian sample is that children may experience more photophobia due to the enhanced mydriatic effect of atropine in less pigmented eyes, resulting in an aversion to spending time outdoors, thus losing the benefits of sunlight on myopia progression. If this theory stands, such as behavioural change as a result of atropine use is likely to be observable in in children in Australia, who are expected to typically spend more time outdoors than those in Singapore, ${ }^{16,17}$ as well as enjoy over 3,200 hours of sunlight annually, compared to approximately 2,000 hours in most parts of East and South East Asia and 1,600 hours in Western Europe.

On the other hand, it is conceivable that atropine and the high levels of sunlight in Perth may work synergistically in slowing myopia progression. If the atropine-induced mydriasis is small and well-tolerated outdoors, it may increase retinal exposure to sunlight and thus slow axial growth, enhancing myopia control. Some researchers ${ }^{40,} 41$ have suggested that atropine "enhanced" the myopia control effects of orthokeratology in a similar way: the mydriasis caused by atropine allowed for more peripheral myopia defocus induced by orthokeratology lenses. However, it 
should be noted that most studies ${ }^{40-42}$ compared a combination atropine-orthokeratology treatment to a monotherapy of only orthokeratology, therefore any myopia control effects in the former group may simply be a result of the atropine treatment alone. Only one retrospective study ${ }^{43}$ directly compared the two, as well as Huang et al.'s meta-analysis, ${ }^{26}$ and both reported that children using atropine have significantly slower myopia progression than those who used orthokeratology. Clearly, there is still much to learn about the efficacy and safety of atropine for myopia control in different populations.

\subsection{I mplications of the study}

The WA-ATOM study is a RCT that will report on the efficacy and safety of low-dose atropine eyedrops for controlling myopia progression in a multi-ethnic cohort of Australian children. Furthermore, the combined data from the WA-ATOM, CHAMP-UK, and MOSAIC studies may allow subgroup analyses based on eye colour, ethnicity, and geographical location. A preliminary secondary analysis may be performed exploring whether time spent outdoors confounds the effects of atropine efficacy on slowing myopia progression. A caveat to the WA-ATOM study is that it is not designed nor powered to explore the optimal period of atropine eyedrop treatment for myopia control, as well as whether there is a need to taper the treatment to minimise or avoid rebound effects. Nonetheless, outcomes from this study will inform clinicians in Australia, and potentially also those in other Western regions, on the appropriateness of prescribing low-dose atropine for myopia control in children. Given the large number of children likely to receive off-label low dose atropine in a non-research setting, efforts should be made to capture information on the efficacy and side effects of ongoing community atropine treatment.

\section{Acknowledgements}


We would like to thank all participants of the WA-ATOM study and their families. We additionally acknowledge the referring optometrists and ophthalmologists from the following practices in Western Australia: SpecSavers Australia, OPSM, Laubman and Pank Optometrists, Spectrum Family Eyecare, Bullcreek Optometrists, Eye5 Optometrists, Options Optometrists, The Optical Superstore, Eyes at Australind, Specs 241, Northam Optical, Cooper and Lourie Optometrists, Crystal Vision Optometrists, Abernethy Owens Optometrists, Future Vision Optometrists, Opticentre, Dr Richard Gardner, Vision Care Centre, Vision West Optometrists, Mr B the Optometrist, Eyecare Centre Carine, Armadale Eyewear, Dr Philip House, Dr Neil Sinclair (Bunbury and Busselton Eye Doctors), Bupa Optical, Dr David de la Hunty, Angelo Street Optical, Eye Focus Optometrists, and EyesWest. We would especially like to thank Ms Nicola Seed, Mrs Magdelena Blaszkowska, Ms Katie Dyer, Dr Feroza J eewa, Mr Nick Stafford-Bell, Dr Michael Richards, and Dr Adrian Koay for assisting in participant recruitment and/or testing. 


\section{REFERENCES}

1. Brodstein RS, Brodstein DE, Olson RJ, Hunt SC, Williams RR. The treatment of myopia with atropine and bifocals. A long-term prospective study. Ophthalmology. 1984;91(11): 1373-9.

2. Clark TY, Clark RA. Atropine 0.01\% Eyedrops Significantly Reduce the Progression of Childhood Myopia. J Ocul Pharmacol Ther. 2015;31(9):541-5. 3. Yen MY, Liu JH, Kao SC, Shiao CH. Comparison of the effect of atropine and cyclopentolate on myopia. Ann Ophthalmol. 1989;21(5):180-2, 7.

4. Kennedy RH, Dyer JA, Kennedy MA, Parulkar S, Kurland LT, Herman DC, et al. Reducing the progression of myopia with atropine: a long term cohort study of Olmsted County students. Binocul Vis Strabismus Q. 2000; 15(3 Suppl):281-304.

5. Gimbel HV. The control of myopia with atropine. Can J Ophthalmol. 1973;8(4):527-32.

6. Chia A, Chua WH, Cheung YB, Wong WL, Lingham A, Fong A, et al. Atropine for the treatment of childhood myopia: safety and efficacy of $0.5 \%, 0.1 \%$, and $0.01 \%$ doses (Atropine for the Treatment of Myopia 2). Ophthalmology. 2012; 119(2):347-54.

7. Chia A, Chua WH, Wen L, Fong A, Goon YY, Tan D. Atropine for the treatment of childhood myopia: changes after stopping atropine $0.01 \%, 0.1 \%$ and $0.5 \%$. Am J Ophthalmol. 2014;157(2):451-7 e1.

8. Chia A, Lu QS, Tan D. Five-Year Clinical Trial on Atropine for the Treatment of Myopia 2: Myopia Control with Atropine 0.01\% Eyedrops. Ophthalmology. 2016;123(2):391-9.

9. Chua WH, Balakrishnan V, Chan YH, Tong L, Ling Y, Quah BL, et al. Atropine for the treatment of childhood myopia. Ophthalmology. 2006; 113(12):2285-91. 
10. Tong L, Huang XL, Koh AL, Zhang X, Tan DT, Chua WH. Atropine for the treatment of childhood myopia: effect on myopia progression after cessation of atropine. Ophthalmology. 2009;116(3):572-9.

11. Yam J C, J iang Y, Tang SM, Law AKP, Chan JJ, Wong E, et al. LowConcentration Atropine for Myopia Progression (LAMP) Study: A Randomized, Double-Blinded, Placebo-Controlled Trial of 0.05\%, 0.025\%, and 0.01\% Atropine Eye Drops in Myopia Control. Ophthalmology. 2018.

12. Yam JC, Li FF, Zhang X, Tang SM, Yip BHK, Kam KW, et al. Two-Year Clinical Trial of the Low-Concentration Atropine for Myopia Progression (LAMP) Study: Phase 2 Report. Ophthalmology. 2019.

13. Holden BA, Fricke TR, Wilson DA, J ong M, Naidoo KS, Sankaridurg P, et al. Global Prevalence of Myopia and High Myopia and Temporal Trends from 2000 through 2050. Ophthalmology. 2016;123(5):1036-42.

14. Salazar M, Shimada K, Patil PN. I ris pigmentation and atropine mydriasis. J Pharmacol Exp Ther. 1976;197(1):79-88.

15. Obianwu HO, Rand MJ . THE RELATIONSHIP BETWEEN THE MYDRIATIC ACTION OF EPHEDRINE AND THE COLOUR OF THE IRIS. The British J ournal of Ophthalmology. 1965;49(5):264-70.

16. Rose KA, Morgan IG, Smith W, Burlutsky G, Mitchell P, Saw SM. Myopia, lifestyle, and schooling in students of Chinese ethnicity in Singapore and Sydney. Arch Ophthalmol. 2008; 126(4):527-30.

17. Read SA, Vincent SJ, Tan CS, Ngo C, Collins MJ, Saw SM. Patterns of Daily Outdoor Light Exposure in Australian and Singaporean Children. Transl Vis Sci Technol. 2018; 7(3):8.

18. McCrann S, Flitcroft I, Strang N, Saunders K, Logan N, Lee S, et al. Myopia Outcome Study of Atropine in Children (MOSAIC): an investigator-led, doublemasked, placebo-controlled, randomised clinical trial protocol [version 2; peer 
review: 2 approved, 1 approved with reservations]. HRB Open Research. 2019;2(15).

19. Azuara-Blanco A, Logan N, Strang N, Saunders K, Allen PM, Weir R, et al. Low-dose $(0.01 \%)$ atropine eye-drops to reduce progression of myopia in children: a multicentre placebo-controlled randomised trial in the UK (CHAMP-UK)-study protocol. Br J Ophthalmol. 2019.

20. Li SM, Wu SS, Kang MT, Liu Y, J ia SM, Li SY, et al. Atropine slows myopia progression more in Asian than white children by meta-analysis. Optom Vis Sci. 2014;91(3):342-50.

21. Bailey IL, Lovie JE. New design principles for visual acuity letter charts. Am J Optom Physiol Opt. 1976;53(11):740-5.

22. Mackey DA, Wilkinson CH, Kearns LS, Hewitt AW. Classification of iris colour: review and refinement of a classification schema. Clin Exp Ophthalmol. 2011;39(5):462-71.

23. Sherwin JC, McKnight CM, Hewitt AW, Griffiths LR, Coroneo MT, Mackey DA. Reliability and validity of conjunctival ultraviolet autofluorescence measurement. $\mathrm{Br}$ J Ophthalmol. 2012;96(6):801-5.

24. Krueger C, Tian L. A comparison of the general linear mixed model and repeated measures ANOVA using a dataset with multiple missing data points. Biol Res Nurs. 2004;6(2):151-7.

25. McCulloch CE, Neuhaus J M. Generalized Linear Mixed Models. Encyclopedia of Biostatistics2005.

26. Huang J, Wen D, Wang Q, McAlinden C, Flitcroft I, Chen H, et al. Efficacy comparison of 16 interventions for myopia control in children: a network metaanalysis. Ophthalmology. 2016;123(4):697-708.

27. Benavente-Perez A, Nour A, Troilo D. Axial eye growth and refractive error development can be modified by exposing the peripheral retina to relative myopic or hyperopic defocus. Invest Ophthalmol Vis Sci. 2014;55(10):6765-73. 
28. Shih YF, Hsiao CK, Chen C, Chang CW, Hung PT, Lin LL. An intervention trial on efficacy of atropine and multi-focal glasses in controlling myopic progression. Acta Ophthalmol Scand. 2001;79(3):233-6.

29. Shih YF, Chen CH, Chou AC, Ho TC, Lin LL, Hung PT. Effects of different concentrations of atropine on controlling myopia in myopic children. J Ocul Pharmacol Ther. 1999; 15(1):85-90.

30. McKnight CM, Sherwin JC, Yazar S, Forward H, Tan AX, Hewitt AW, et al. Myopia in young adults is inversely related to an objective marker of ocular sun exposure: the Western Australian Raine cohort study. Am J Ophthalmol. 2014;158(5):1079-85.

31. Rose KA, Morgan IG, Ip J, Kifley A, Huynh S, Smith W, et al. Outdoor activity reduces the prevalence of myopia in children. Ophthalmology. 2008;115(8):1279-85. 32. Rose KA, French AN, Morgan IG. Environmental Factors and Myopia: Paradoxes and Prospects for Prevention. Asia Pac J Ophthalmol (Phila). 2016;5(6):403-10.

33. Xiong S, Sankaridurg P, Naduvilath T, Zang J, Zou H, Zhu J, et al. Time spent in outdoor activities in relation to myopia prevention and control: a meta-analysis and systematic review. Acta Ophthalmol. 2017;95(6):551-66.

34. Sherwin JC, Reacher MH, Keogh RH, Khawaja AP, Mackey DA, Foster PJ. The association between time spent outdoors and myopia in children and adolescents: a systematic review and meta-analysis. Ophthalmology. 2012;119(10):2141-51.

35. McBrien NA, Moghaddam HO, Reeder AP. Atropine reduces experimental myopia and eye enlargement via a nonaccommodative mechanism. Invest Ophthalmol Vis Sci. 1993;34(1):205-15.

36. Gallego P, Martinez-Garcia C, Perez-Merino P, I bares-Frias L, Mayo-Iscar A, Merayo-Lloves J. Scleral changes induced by atropine in chicks as an experimental model of myopia. Ophthalmic Physiol Opt. 2012;32(6):478-84. 
37. Barathi VA, Chaurasia SS, Poidinger M, Koh SK, Tian D, Ho C, et al. Involvement of GABA transporters in atropine-treated myopic retina as revealed by iTRAQ quantitative proteomics. J Proteome Res. 2014;13(11):4647-58.

38. Stone RA, Liu J, Sugimoto R, Capehart C, Zhu X, Pendrak K. GABA, experimental myopia, and ocular growth in chick. Invest Ophthalmol Vis Sci. 2003;44(9):3933-46.

39. Arumugam B, McBrien NA. Muscarinic antagonist control of myopia: evidence for M4 and M1 receptor-based pathways in the inhibition of experimentally-induced axial myopia in the tree shrew. Invest Ophthalmol Vis Sci. 2012;53(9):5827-37. 40. Chen Z, Huang S, Zhou J, Xiaomei Q, Zhou X, Xue F. Adjunctive effect of orthokeratology and low dose atropine on axial elongation in fast-progressing myopic children-A preliminary retrospective study. Cont Lens Anterior Eye. 2018. 41. Wan L, Wei CC, Chen CS, Chang CY, Lin CJ, Chen JJ, et al. The Synergistic Effects of Orthokeratology and Atropine in Slowing the Progression of Myopia. J Clin Med. 2018; 7(9).

42. Kinoshita N, Konno Y, Hamada N, Kanda Y, Shimmura-Tomita M, Kakehashi A. Additive effects of orthokeratology and atropine $0.01 \%$ ophthalmic solution in slowing axial elongation in children with myopia: first year results. J pn J Ophthalmol. 2018;62(5):544-53.

43. Lin HJ, Wan L, Tsai FJ, Tsai YY, Chen LA, Tsai AL, et al. Overnight orthokeratology is comparable with atropine in controlling myopia. BMC Ophthalmol. 2014; 14:40. 


\section{University Library}

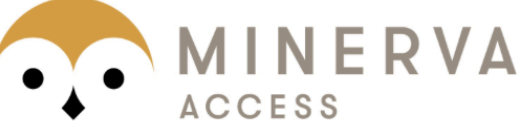

A gateway to Melbourne's research publications

Minerva Access is the Institutional Repository of The University of Melbourne

Author/s:

Lee, SSY;Mackey, DA;Lingham, G;Crewe, JM;Chen, FK;Charng, J;Ng, F;Flitcroft,

I;Loughman, JJ;Azuara-Blanco, A;Logan, NS;Hammond, CJ;Chia, A;Richards, MD;Truong,

TT;Clark, A

Title:

Western Australia Atropine for the Treatment of Myopia (WA-ATOM) study: Rationale, methodology and participant baseline characteristics

Date:

2020-04-01

Citation:

Lee, S. S. Y., Mackey, D. A., Lingham, G., Crewe, J. M., Chen, F. K., Charng, J., Ng, F., Flitcroft, I., Loughman, J. J., Azuara-Blanco, A., Logan, N. S., Hammond, C. J., Chia, A., Richards, M. D., Truong, T. T. \& Clark, A. (2020). Western Australia Atropine for the Treatment of Myopia (WA-ATOM) study: Rationale, methodology and participant baseline characteristics. CLINICAL AND EXPERIMENTAL OPHTHALMOLOGY, 48 (5), pp.569-579. https://doi.org/10.1111/ceo.13736.

Persistent Link:

http://hdl.handle.net/11343/275592 